\title{
Nanowire Characterization for Photonic Properties
}

\author{
David C. Bell, ${ }^{1,3}$ Carl J. Barrelet ${ }^{2}$, Silvija Gradečak ${ }^{2}$ and Charles M. Lieber ${ }^{2,3}$ \\ ${ }^{1}$ Center for Imaging and Mesoscale Systems \\ ${ }^{2}$ Department of Chemistry and Chemical Biology \\ ${ }^{3}$ Division of Engineering and Applied Sciences \\ Harvard University, Cambridge, MA 02138
}

Single-source molecular precursors [1] have been used to synthesize single crystalline cadmium sulfide (CdS) nanowires. CdS nanowires can act as waveguides and exhibit distinct properties from conventional transparent dielectric waveguides since absorption and emission occur for modes with near band edge energies. [2] Combined structural and optical characterization is the key to understanding these materials for use in photonic applications. Analytical electron microscopy methods have included, Energy Dispersive X-Ray Spectroscopy (EDS), (CL), Electron Energy LossSpectroscopy (EELS) and photoluminescence spectroscopy (PL) on a single nanowire. Discrete small volume microanalysis is required for complete materials characterization. Nanowires with compositions of $\mathrm{ZnS}$ and $\mathrm{GaN}$ have also been examined.

$\mathrm{CdS}$ is a direct band-gap semiconductor with useful and functional photonic properties. Critical to the function of the material is the stoichiometry and morphology of the synthesized nanowires. Morphology can be determined by the transmission electron microscopy (TEM) [3]. Nanowires approaching uniform diameter of $10 \mathrm{~nm}$ have been produced from a corresponding gold nanocluster (Figure 1). EDS has been used to determine the compositions of the nanowires with typical compositions of order $\mathrm{Cd} 50.7 \%$ and $\mathrm{S} 49.3 \%$ (Figure 2).

Cathodoluminescence ttechniques are very useful for the quantitative and spatially-resolved analysis of optical properties of semiconductors general, and for monitoring the effects related to their growth process. Residual stresses and impurities can strongly affect the waveguiding properties of the nanowires because, as a consequence of the stress-optic effect, the waveguide can be changed and transmission losses increased. The optical properties of the nanowires have been examined by photoluminescence and can be used as a representation of waveguide quality. A typical photoluminescence spectrum obtained form a nominal $25 \mathrm{~nm}$ diameter nanowire shows a single peak centred at $513 \mathrm{~nm}$ and a corresponding full width half maximum of $21 \mathrm{~nm}$ (Figure 3). The narrow peak in the photoluminescence spectrum being an indication of the well defined optical properties of the nanowire, linked directly to the single crystal structure. Narrowly defined optical properties are essential for the use of these nanowires as building blocks for the construction of next generation photonic devices. Characterization of their optical properties is critical for the future development of photonic nanowires.

\section{References}

[1] C. J. Barrelet, Y. Wu, D. C. Bell, C. M. Lieber, J. Am. Chem. Soc. 125, 11498-11499 (2003)

[2] C. J. Barrelet, A. B. Greytak, C. M. Lieber, Nanoletters 4, 10 1981-1985 (2004)

[3] D. C. Bell et al, Microsc. Res. Techniq. 64, 373-389 (2004) 


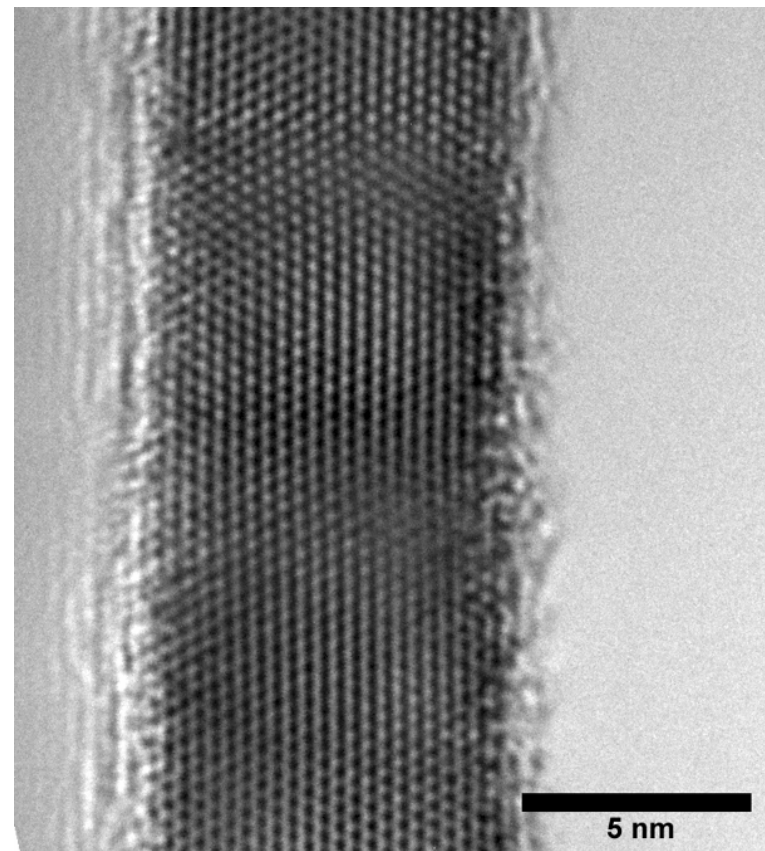

Figure 1. High resolution TEM image of a CdS nanowire of nominal $9 \mathrm{~mm}$ diameter

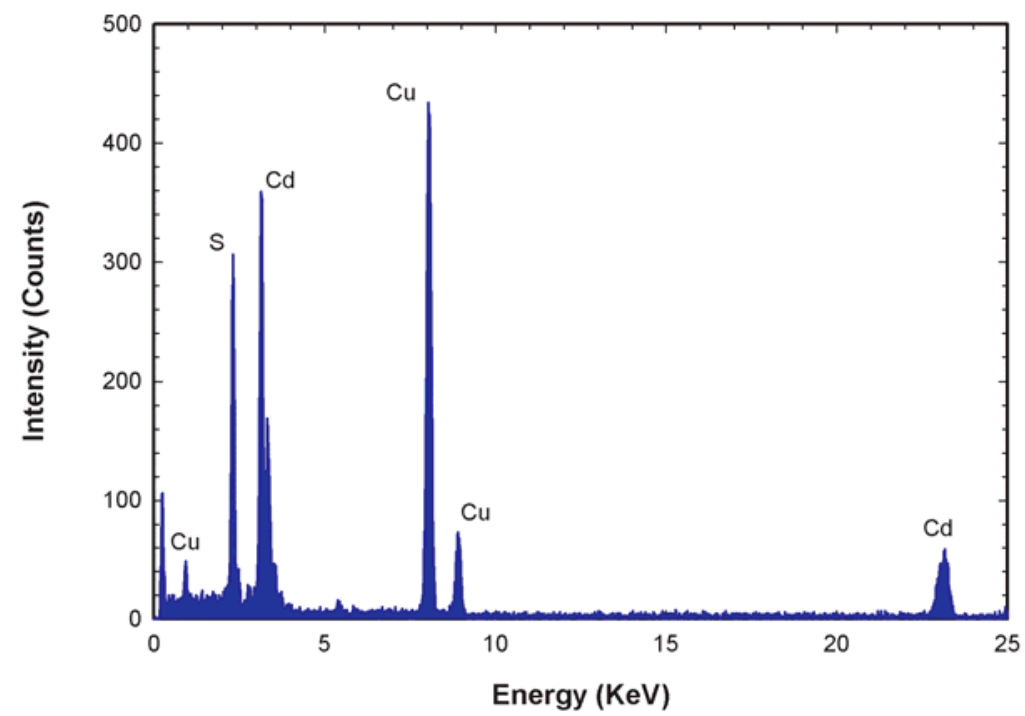

Figure 2. Energy dispersive $\mathrm{x}$-ray spectrum from a single $\mathrm{CdS}$ nanowire (Cu Peak from grid)

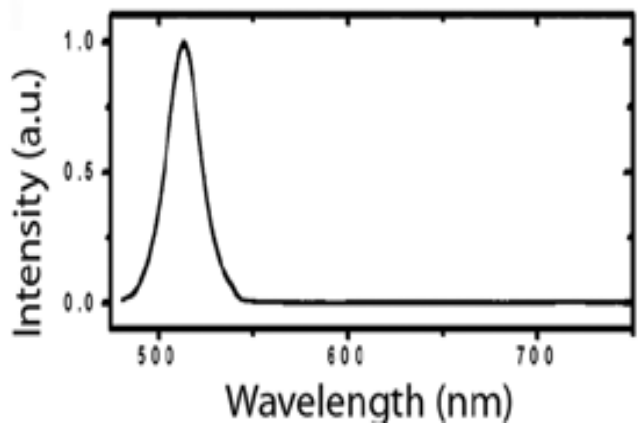

Figure 3. Photoluminescence spectrum from a single $25 \mathrm{~nm}$ diameter $\mathrm{CdS}$ nanowire, (excited at $400 \mathrm{~nm})$. 